\title{
Femtosecond photoelectron diffraction on laser-aligned molecules: Towards time-resolved imaging of molecular structure
}

\author{
R. Boll, ${ }^{1,2,3, *}$ D. Anielski, ${ }^{1,2,3}$ C. Bostedt, ${ }^{4}$ J. D. Bozek, ${ }^{4}$ L. Christensen, ${ }^{5}$ R. Coffee, ${ }^{4}$ S. De,${ }^{5,6}$ \\ P. Decleva, ${ }^{7}$ S. W. Epp, ${ }^{1,2,8}$ B. Erk, ${ }^{1,2,3}$ L. Foucar, ${ }^{1,9}$ F. Krasniqi, ${ }^{1,8,9}$ J. Küpper, ${ }^{10,11,12}$ \\ A. Rouzée, ${ }^{13,14}$ B. Rudek, $, 2,3,15$ A. Rudenko, ${ }^{1,2,16}$ S. Schorb, ${ }^{4}$ H. Stapelfeldt, ${ }^{17}$ \\ M. Stener, ${ }^{7}$ S. Stern, ${ }^{10,11}$ S. Techert,${ }^{3,18,19}$ S. Trippel, ${ }^{10}$ M. J. J. Vrakking, ${ }^{13,14}$ J. Ullrich, ${ }^{1,2,15}$ and D. Rolles ${ }^{1,3,9, \dagger}$ \\ ${ }^{1}$ Max Planck Advanced Study Group at CFEL, 22607 Hamburg, Germany \\ ${ }^{2}$ Max Planck Institute for Nuclear Physics, 69117 Heidelberg, Germany \\ ${ }^{3}$ Deutsches Elektronen Synchrotron (DESY), 22607 Hamburg, Germany \\ ${ }^{4}$ SLAC National Accelerator Laboratory, Menlo Park, California 94025, USA \\ ${ }^{5}$ Department of Physics and Astronomy, Aarhus University, 8000 Aarhus C, Denmark \\ ${ }^{6}$ Saha Institute of Nuclear Physics, 700064 Kolkata, India \\ ${ }^{7}$ Dipartimento di Scienze Chimiche e Farmaceutiche, Università di Trieste, 34127 Trieste, Italy \\ ${ }^{8}$ Max Planck Institute for Structural Dynamics, 2607 Hamburg, Germany \\ ${ }^{9}$ Max Planck Institute for Medical Research, 69120 Heidelberg, Germany \\ ${ }^{10}$ Center for Free-Electron Laser Science, DESY, 22607 Hamburg, Germany \\ ${ }^{11}$ Department of Physics, University of Hamburg, 22761 Hamburg, Germany \\ ${ }^{12}$ Center for Ultrafast Imaging, University of Hamburg, 22761 Hamburg, Germany \\ ${ }^{13}$ Max-Born-Institut, 12489 Berlin, Germany \\ ${ }^{14}$ FOM-Institute AMOLF, 1098 XG Amsterdam, The Netherlands \\ ${ }^{15}$ Physikalisch-Technische Bundesanstalt (PTB), 38116 Braunschweig, Germany \\ ${ }^{16}$ J.R. MacDonald Laboratory, Department of Physics, Kansas State University, Manhattan, Kansas 66506, USA \\ ${ }^{17}$ Department of Chemistry, Aarhus University, 8000 Aarhus C, Denmark \\ ${ }^{18}$ Max Planck Institute for Biophysical Chemistry, 37077 Göttingen, Germany \\ ${ }^{19}$ Institute of X-ray Physics, 37077 Göttingen University, Germany
}

(Received 6 August 2013; published 6 December 2013)

\begin{abstract}
We demonstrate an experimental method to record snapshot diffraction images of polyatomic gas-phase molecules, which can, in a next step, be used to probe time-dependent changes in the molecular geometry during photochemical reactions with femtosecond temporal and angstrom spatial resolution. Adiabatically laser-aligned 1-ethynyl-4-fluorobenzene $\left(\mathrm{C}_{8} \mathrm{H}_{5} \mathrm{~F}\right)$ molecules were imaged by diffraction of photoelectrons with kinetic energies between 31 and $62 \mathrm{eV}$, created from core ionization of the fluorine $(1 s)$ level by $\approx 80 \mathrm{fs}$ x-ray free-electronlaser pulses. Comparison of the experimental photoelectron angular distributions with density functional theory calculations allows relating the diffraction images to the molecular structure.
\end{abstract}

In the past two decades, our understanding of chemical reactions including the occurrence of transition states and the dynamics in the vicinity of conical intersections has advanced drastically due to substantial progress in quantum chemistry calculations and in the field of femtochemistry [1]. Observing the ultrafast movement of atoms and electrons during a chemical reaction experimentally is extremely challenging due to the molecular dimensions of typically only a few angstroms and reaction time scales which are often on the order of femtoseconds. Such spatiotemporal resolution can nowadays be provided by the pulses of a free-electron laser (FEL) [2,3], and femtosecond x-ray diffraction has already been successfully demonstrated for large biomolecules and nano-objects [4-6]. For small gas-phase molecules, it suffers from very low elastic scattering cross sections, making it difficult to achieve sufficiently good signal-to-noise ratios and effectively limiting it to targets containing heavy atomic constituents [7].

\footnotetext{
*rebecca.boll@asg.mpg.de

†daniel.rolles@desy.de
}

A way to overcome this difficulty is to image the molecules with electrons instead of photons [8]: A $7 \mathrm{keV} \mathrm{x}$-ray with a wavelength of $1.75 \AA$ has an elastic scattering cross section of $<10 \mathrm{~b}$ on a carbon atom [9]. An electron with a kinetic energy of $50 \mathrm{eV}$ has the same wavelength but an elastic cross section of $470 \mathrm{Mb}$ [10]. However, electron beams are hard to compress to short, intense pulses and it has been demonstrated only recently that pulse durations below 100 fs can be achieved for pulses of $10^{6}$ electrons [11]. Moreover, laser-pump electron-probe measurements on extended targets such as molecular beams suffer from velocity mismatch between the two pulses which has, so far, limited their overall achievable temporal resolution to $>850$ fs [12-14].

Our approach, which exploits the advantages of an electron probe pulse while avoiding the velocity mismatch, is to image the molecules from the inside with photoelectrons that are created directly at a specific atom within the molecule. In contrast to related experiments using time-resolved valence photoionization [15], which probe mostly the electronic structure, photoelectron diffraction with localized inner-shell electrons can directly image the geometric structure [16]. It is a well-established technique for structure determination 
of solids, surfaces, and adsorbates [17-19], offering the possibility to image only the local environment of an emitter due to element-specific core ionization. It can also be applied to gas-phase molecules, where the emitted photoelectron wave is modulated by interference from intramolecular scattering on neighboring atoms that contains the information on the molecular structure [16,20-22].

In order to be able to extract this information from the recorded photoelectron angular distributions, the reference frame of the molecule has to be known. A priori, this is not the case for an ensemble of randomly oriented gasphase molecules. Avoiding the averaging over all possible orientations can be achieved in two ways: The molecular axes can be determined from the measurement a posteriori, for example in an angle-resolved photoelectron-photoion coincidence experiment [20-24], or the molecules can be actively aligned prior to the photoionization, for example by impulsive or adiabatic laser alignment [25]. The former allows probing the molecules in a field-free environment and can provide a high degree of alignment. However, it is applicable only for rather small molecules and requires less than one ionization event per pulse, thus severely limiting the amount of data that can be collected given the repetition rates of the currently operating $\mathrm{x}$-ray FELs $(<120 \mathrm{~Hz})$. We have, therefore, chosen to employ adiabatic laser alignment, which allows imaging a whole ensemble of molecules with each $\mathrm{x}$-ray pulse.

We present here an application of the FEL-based femtosecond photoelectron diffraction method to polyatomic gas-phase molecules. The recorded diffraction patterns can be interpreted as freeze frames of a molecular movie which can be extended to a complete movie by following the temporal changes of the molecular geometry in a pump-probe experiment.

In the present case, we were imaging 1-ethynyl-4fluorobenzene molecules (pFAB) (see Fig. 1), with photoelectrons at five different kinetic energies between 31 and $62 \mathrm{eV}$. pFAB was chosen as a prototype system, because the photochemistry of several related phenyl halides has been studied previously and the existence of several shortlived transition states has been suggested, for example for fluorobenzene [26,27].

The experiment was performed at the AMO beamline of the Linac Coherent Light Source (LCLS) at SLAC National Accelerator Laboratory [28] using the CFEL-ASG MultiPurpose (CAMP) end station [29]. The data were analyzed with the CFEL-ASG software suite (CASS) [30]. The experimental setup is depicted in Fig. 1. pFAB molecules seeded in helium (50 bars backing pressure) were introduced into the CAMP chamber as a rotationally cold $(\approx 1 \mathrm{~K})$, pulsed molecular beam produced by supersonic expansion from an Even-Lavie valve (nozzle diameter $150 \mu \mathrm{m}$ ). The coldest molecules in the beam were selected by an electrostatic deflector [31]. The molecular beam was then crossed by a nonionizing Nd:YAG (yttrium aluminum garnet) laser (Spectra Physics Quanta Ray Pro, $1064 \mathrm{~nm}, 10 \mathrm{~ns}$ pulse length, $500 \mathrm{~mJ}$ pulse energy), which was propagated collinearly with the FEL and induced adiabatic, one-dimensional alignment of the $\mathrm{F}-\mathrm{C}$ bond axis along the laser polarization. At the time of maximum alignment in the peak of the Nd:YAG pulse, the FEL pulse (723-754 eV photon energy, $0.2 \%-1.0 \%$ bandwidth [2], $\approx 80 \mathrm{fs}$ pulse length,

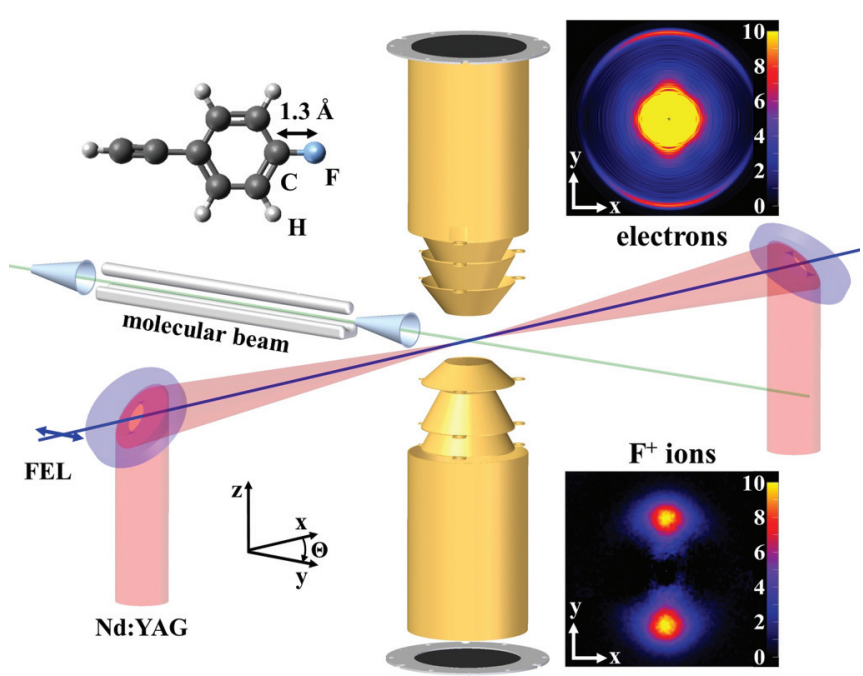

FIG. 1. (Color online) Experimental setup: pFAB molecules were introduced into the CAMP chamber as a cold, pulsed molecular beam (green) after passing through two skimmers and an electrostatic deflector. A Nd:YAG laser (red) induced adiabatic, one-dimensional alignment of the molecular ensemble, which was then hit by an X-ray pulse (blue). The resulting photoelectrons and fragment ions were extracted towards two MCP-phosphor screen detectors by the electric field of a velocity map imaging spectrometer. The detector images present typical electron (top) and $\mathrm{F}^{+}$-ion distributions (bottom) for aligned molecules after background subtraction. The polarization axes of the Nd:YAG laser and the FEL were parallel and along the $y$ axis as indicated by the blue arrow.

0.6-1.2 mJ pulse energy) hit the ensemble of molecules, ionizing predominantly the fluorine $(1 s)$ shell and resulting in photoelectrons with kinetic energies of 31-62 eV [the $\mathrm{F}(1 s)$ binding energy in fluorobenzene is $692 \mathrm{eV}$ [32]]. The beam waist of the Nd:YAG laser $\left(\omega_{0} \approx 60 \mu \mathrm{m}\right)$ was chosen larger than that of the FEL $\left(\omega_{0} \approx 40 \mu \mathrm{m}\right)$ in order to assure that all molecules probed by the FEL were aligned.

Electrons and ionic fragments produced by the FEL pulses were accelerated by the inhomogeneous electric field of a double-sided velocity map imaging spectrometer (VMI) and were detected simultaneously on two microchannel plate (MCP) detectors with phosphor screens, which were imaged with a 1 megapixel CCD camera that was read out for every single FEL shot. The VMI voltages were chosen such that the $\mathrm{F}(1 s)$ photoelectrons and all fragment ions were collected in the full $4 \pi$ solid angle. By fast switching of the high voltage, the electron detector was gated to suppress stray electrons and the ion detector was gated to record only ions with a given mass-to-charge ratio.

The simultaneous detection of electrons and ions allowed constant monitoring of the degree of alignment by analyzing the angular distribution of a characteristic fragment ion. In the present case, we monitored the $\mathrm{F}^{+}$ions which are emitted along the $\mathrm{F}-\mathrm{C}$ bond direction. The resulting detector image is shown in Fig. 1. The achieved degree of alignment can be characterized by an alignment parameter [25] of $\left\langle\cos ^{2} \theta\right\rangle_{2 \mathrm{D}}=$ 0.85 . An isotropic background of lower energy fragments has been subtracted, which stems from clusters in the molecular beam. 
The electron image for aligned molecules recorded at a photon energy of $743 \mathrm{eV}$ is also shown in Fig. 1. It was obtained by inversion of the raw VMI image using the pBasex algorithm [33]. Because of the cylindrical symmetry of the photoelectron angular distribution around the polarization axis of the FEL and the Nd:YAG laser, which are parallel and lie in the detector plane, this allows reconstruction of the three-dimensional photoelectron distribution from its twodimensional projection. The $\mathrm{F}(1 s)$ photoline with an electron kinetic energy of $51 \mathrm{eV}$ is clearly visible as a sharp ring at large radii. The low-energy electrons in the center of the image are mainly created by shake-off and shake-up processes. High-energy electrons, for example from $\mathrm{C}(1 s)$ and valence ionization as well as Auger electrons, have kinetic energies $>240 \mathrm{eV}$. They are thus collected in a small solid angle for the chosen spectrometer voltages and appear only as a small background.

At first sight, the $\mathrm{F}(1 s)$ photoline shows surprisingly little angular structure from interference and displays an essentially " $p$-wave"-like angular distribution along the $\mathrm{x}$-ray polarization axis that is typical for $(1 s)$ ionization. One reason for this is the fact that for the achieved degree of alignment the F-C axes were only confined to an opening angle of $40^{\circ}$ (FWHM), which significantly smears out the interference minima and maxima. Additionally, a background of unaligned molecules from pFAB clusters in the beam further reduces the contrast. To extract the intensity variations caused by intramolecular scattering of the photoelectrons, we therefore consider in the following the differences between the VMI images obtained with and without the Nd:YAG alignment laser, which were recorded concurrently by running the LCLS at $120 \mathrm{~Hz}$, the molecular beam at $60 \mathrm{~Hz}$, and the Nd:YAG laser at $30 \mathrm{~Hz}$.

The resulting difference images recorded at five photon energies corresponding to photoelectron kinetic energy from 31 to $62 \mathrm{eV}$ are shown in Fig. 2. Here, the photoelectron intensity on the circular detector is shown as a color map

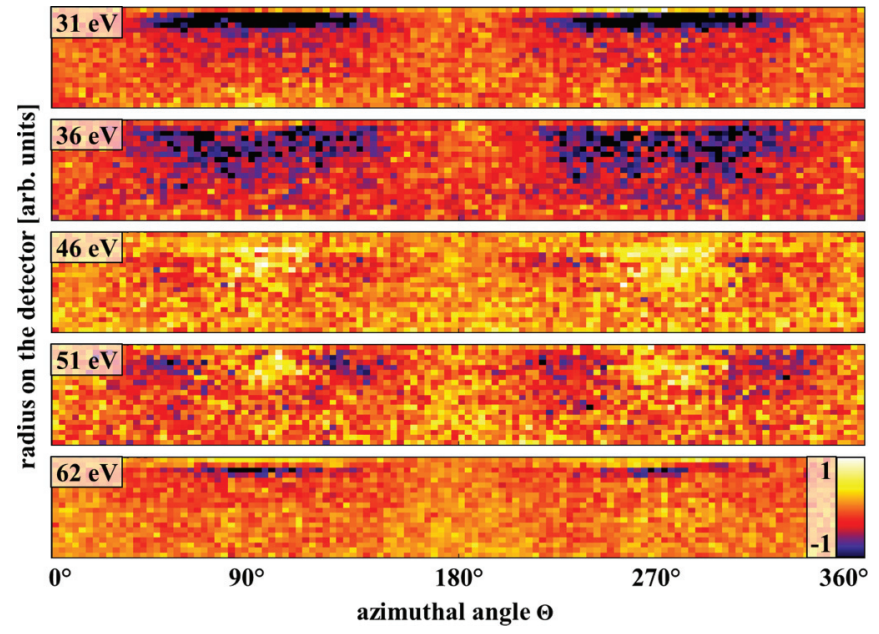

FIG. 2. (Color online) Difference between the raw electron VMI images obtained with and without the Nd:YAG alignment laser for five different photon energies. Only the radius region on the detector that corresponds to the fluorine $(1 s)$ photoline is shown as a function of the azimuthal detection angle. The polarization axes of the Nd:YAG laser and FEL are at $90^{\circ} / 270^{\circ}$ in Cartesian coordinates with the abscissa being the distance from the center of the VMI image (i.e., the radius coordinate, which contains information on both the photoelectron energy and the polar emission angle) and the ordinate being the azimuthal angle on the detector. The difference images show pronounced angular structures which vary as a function of photon energy and which can be attributed to photoelectron diffraction effects. The chosen electron energies correspond to de Broglie wavelengths of 1.53 to $2.16 \AA$, which are comparable to the internuclear distances in the molecule, as the F-C distance in pFAB is $1.3 \AA$ and the overall length of the molecule is $4.3 \AA$.

We can now investigate the diffraction effects quantitatively by radially integrating the difference images in Fig. 2. The resulting photoelectron angular distribution differences ( $\triangle$ PADs) are shown in Fig. 3 as polar plots. They are compared to the $\triangle$ PADs obtained by subtracting the corresponding inverted VMI images. Both raw and inverted data agree well within the statistical uncertainties, thus confirming the reliability of the inversion procedure used in our analysis.

In order to interpret the observed effects and to further elucidate their relation to the molecular structure, we have performed density functional theory (DFT) calculations with a linear combination of atomic orbitals $B$-spline code that has already been used previously to calculate accurate cross sections and molecular-frame photoelectron angular distributions for small polyatomic molecules [34-36]. The experimentally achieved degree of molecular alignment is accounted for in the calculations by averaging over a two-dimensional Gaussian with a FWHM of $40^{\circ}$. The calculated photoelectron angular distributions for aligned and randomly oriented molecules are then normalized to the integrated signal in the corresponding experimental photoelectron angular distributions and subtracted. The resulting calculated $\triangle$ PADs are shown in Fig. 3 as dotted lines. They are in good agreement with the experimental data.

Based on our calculations, we identify two contributions to the energy dependence of the $\triangle$ PADs: Most prominent is a change of the sign of the integral difference, which is positive for $46 \mathrm{eV}$ and negative for all other photoelectron energies. This change can be explained by a difference of the partial ionization cross sections for molecules aligned parallel to the $\mathrm{x}$-ray polarization axis as compared to randomly oriented molecules, both of which vary as a function of photon energy [37]. This effect can be used to calibrate the energy offset between experiment and calculation. Such an offset is well known to occur since the LB94 exchange correlation potential employed in the DFT calculations is too attractive, which causes a shift of the cross sections to lower binding energies [38].

The second contribution to the $\triangle$ PADs is due to the angular redistribution of photoelectron intensity because of intramolecular scattering. The dominant feature is a stronger confinement of the electron emission towards the direction of the nearest-neighbor atom which is situated on the aligned molecular axis (see arrow), as can be seen most clearly for $46 \mathrm{eV}$. This is commonly referred to as the "forward scattering peak" in photoelectron diffraction [17-19]. Further structure results from interference maxima and minima whose angular positions vary as a function of electron wavelength. 


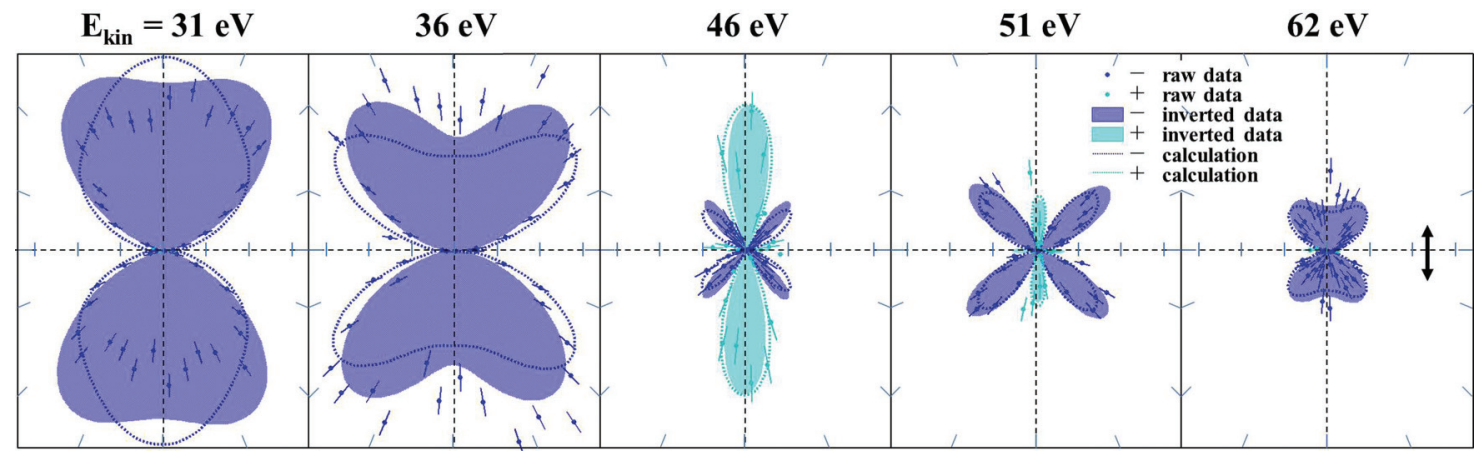

FIG. 3. (Color online) Fluorine $1 s$ photoelectron angular distribution differences ( $\triangle$ PADs) as a function of photoelectron kinetic energy in a polar representation. Positive values of the difference $(+)$ are plotted in cyan, negative values $(-)$ in blue. The experimental differences were calculated by subtracting the $\mathrm{F}(1 s)$ photoelectron angular distributions obtained without Nd:YAG laser from those obtained with Nd:YAG, each normalized to the number of corresponding FEL shots. Raw (dots) and inverted data (shaded areas) are compared to the differences obtained from DFT calculations (dotted lines), which take into account the experimentally achieved degree of alignment as well as the background from unaligned molecules due to clusters in the molecular beam. In order to account for an energy offset between experiment and theory, the energy scale of the calculations was shifted higher by $10 \mathrm{eV}$ based on the comparison of measured and calculated partial cross sections (see text). The polarization axes of alignment laser and FEL were parallel and are indicated by the arrow.

Since this interference depends on both the wavelength and the geometry of the scattering object, the change of the $\triangle$ PADs for decreasing wavelengths gives an indication of the changes expected when a molecule with increasing internuclear distances, for example during a photodissociation, is imaged at a fixed wavelength.

The direct relation of the photoelectron diffraction to the molecular structure as well as the potential of the method for time-resolved imaging is illustrated by comparing the calculated molecular-frame photoelectron angular distributions for different molecular geometries shown in Fig. 4. Only the positions of the fluorine atom and of one adjacent hydrogen atom are varied, which causes significant changes in the angular distributions. The geometries chosen for these calculations are based on different transition states which are predicted to occur within $<1$ ps after photoexcitation of fluorobenzene by a UV photon at $193 \mathrm{~nm}$ [26,27]. With the femtosecond photoelectron diffraction approach presented

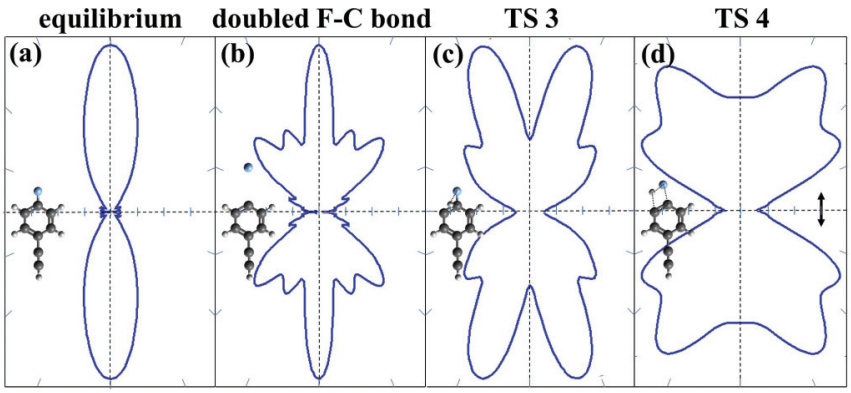

FIG. 4. (Color online) Calculated fluorine $(1 s)$ photoelectron angular distributions for $\mathrm{pFAB}$ at a photoelectron kinetic energy of $35 \mathrm{eV}$ obtained from DFT calculations for (a) the equilibrium geometry, (b) for a pFAB molecule with twice the equilibrium bond length between fluorine and the benzene ring, and [(c), (d)] for two proposed transition states TS 3 and TS 4 [27]. The molecules are one-dimensionally aligned with the F-C axis parallel to the $\mathrm{x}$-ray polarization as indicated by the arrow. The calculations correspond to an experimental kinetic energy of $45 \mathrm{eV}$. here, it should be possible to image the reaction pathway through one of these transition states directly in a UV-pump, FEL-probe experiment.

In summary, our results demonstrate the feasibility of femtosecond photoelectron diffraction on polyatomic gasphase molecules by measuring the photoelectron angular distributions of laser-aligned molecules at an x-ray freeelectron laser. We observe pronounced changes for varying photon energies and thus varying photoelectron wavelengths, which are well described by DFT calculations demonstrating the sensitivity of the method to the molecular structure on an angstrom scale. Femtosecond photoelectron diffraction therefore has the potential to become a versatile tool to study, in particular, short-lived molecular structures provided they contain a well-defined emitter atom with a localized electronic orbital from which the primary electron wave can be emitted. Furthermore, although the laser-alignment technique chosen here is certainly not applicable to all molecules, it applies to a broad range of gas-phase molecules whose polarizability tensor is anisotropic. In addition, for suitable classes of molecules, it will also be possible to determine, very precisely, the molecular orientation without any prealignment by using electron-ion coincidence techniques once higher repetitionrate FEL sources (such as the European XFEL) are available. When taking time-dependent diffraction patterns of a changing molecular geometry in a pump-probe setup, the technique presented here allows observing geometrical changes during a chemical reaction in real time. With FEL pulse lengths of less than $10 \mathrm{fs}$, available today for example at LCLS, and by employing cross-correlation techniques to accurately determine the jitter between laser-pump and FEL-probe pulses $[39,40]$, the achievable time resolution can be on the order of $30 \mathrm{fs}$, limited currently by the length of the laser-pump pulse.

Part of this research was carried out at the Linac Coherent Light Source (LCLS) at the SLAC National Accelerator Laboratory. LCLS is an Office of Science User Facility operated for the US Department of Energy Office of Science by Stanford University. Additional measurements 
were performed at DORIS (DESY) at beamline BW3. We acknowledge the Max Planck Society for funding the development and operation of the CAMP instrument within the ASG at CFEL. D.R. acknowledges support from the Helmholtz Gemeinschaft through the Young Investigator Program. L.C., S.D., and H.S. acknowledge support from the Carlsberg Foundation. J.K. and S.Tr. acknowledge support from the excellence cluster The Hamburg Centre for Ultrafast Imaging-Structure, Dynamics and Control of Matter at the Atomic Scale of the Deutsche Forschungsgemeinschaft. A.Ro. acknowledges the research program of the "Stichting voor Fundamenteel Onderzoek der Materie," which is financially supported by the "Nederlandse organisatie voor Wetenschappelijk Onderzoek." A.Ru. acknowledges support from the Chemical Sciences, Geosciences, and Biosciences Division, Office of Basic Energy Sciences, Office of Science, US Department of Energy. S.Te. acknowledges support through SFB 755 Nanoscale photonic imaging. We are grateful to F. Filsinger, L. Holmegaard, P. Johnsson, and J. Thøgersen for help in preparing the molecular beam and laser-alignment setup. We also thank T. Delmas, L. Gumprecht, A. Hömke, C. Schmidt, and M. Swiggers for technical support and the LCLS staff for their support and hospitality during the beam times.
[1] A. H. Zewail, J. Phys. Chem. A 104, 5660 (2000).

[2] P. Emma et al., Nat. Photonics 4, 641 (2010).

[3] T. Ishikawa et al., Nat. Photonics 6, 540 (2012).

[4] H. N. Chapman et al., Nature (London) 470, 73 (2011).

[5] T. Gorkhover et al., Phys. Rev. Lett. 108, 245005 (2012).

[6] A. Barty, J. Küpper, and H. N. Chapman, Annu. Rev. Phys. Chem. 64, 415 (2013).

[7] J. Küpper et al., arXiv:1307.4577v1.

[8] M. Chergui and A. H. Zewail, ChemPhysChem 10, 28 (2009).

[9] X-Ray Data Booklet, edited by A. C. Thompson, Rev. 3 (LBNL/PUB-490, Berkeley, 2009), http://xdb.lbl.gov/.

[10] NIST electron elastic-scattering cross-section database, Version 3.2 (2013).

[11] T. van Oudheusden, P. L. E. M. Pasmans, S. B. van der Geer, M. J. de Loos, M. J. van der Wiel, and O. J. Luiten, Phys. Rev. Lett. 105, 264801 (2010).

[12] H. Ihee, V. A. Lobastov, U. M. Gomez, B. M. Goodson, R. Srinivasan, C.-Y. Ruan, and A. H. Zewail, Science 291, 458 (2001).

[13] G. Sciaini and R. J. D. Miller, Rep. Prog. Phys. 74, 096101 (2011).

[14] C. Hensley, J. Yang, and M. Centurion, Phys. Rev. Lett. 109, 133202 (2012).

[15] F. Kelkensberg, A. Rouzée, W. Siu, G. Gademann, P. Johnsson, M. Lucchini, R. R. Lucchese, and M. J. J. Vrakking, Phys. Rev. A 84, 051404(R) (2011).

[16] F. Krasniqi, B. Najjari, L. Strüder, D. Rolles, A. Voitkiv, and J. Ullrich, Phys. Rev. A 81, 033411 (2010).

[17] C. S. Fadley, in Synchrotron Radiation Research, edited by Robert Z. Bachrach (Plenum Press, New York, 1992).

[18] D. P. Woodruff and A. M. Bradshaw, Rep. Prog. Phys. 57, 1029 (1994).

[19] C. S. Fadley, Surf. Interface Anal. 40, 1579 (2008).

[20] U. Becker, O. Gessner, and A. Rüdel, J. Electron Spectrosc. 108, 189 (2000).
[21] A. Landers et al., Phys. Rev. Lett. 87, 013002 (2001).

[22] B. Zimmermann et al., Nat. Phys. 4, 649 (2008).

[23] A. Yagishita, K. Hosaka, and J.-I. Adachi, J. Electron Spectrosc. 142, 295 (2005).

[24] K. L. Reid, Mol. Phys. 110, 131 (2012).

[25] H. Stapelfeldt and T. Seideman, Rev. Mod. Phys. 75, 543 (2003).

[26] C. L. Huang, J. C. Jiang, A. M. Mebel, Y. T. Lee, and C. K. Ni, J. Am. Chem. Soc. 125, 9814 (2003).

[27] C.-Y. Wu, Y.-J. Wu, and Y.-P. Lee, J. Chem. Phys. 121, 8792 (2004).

[28] C. Bostedt et al., J. Phys. B 46, 164003 (2013).

[29] L. Strüder et al., Nucl. Instrum. Methods Phys. Res., Sect. A 614, 483 (2010).

[30] L. Foucar et al., Comput. Phys. Commun. 183, 2207 (2012).

[31] F. Filsinger, J. Küpper, G. Meijer, L. Holmegaard, J. H. Nielsen, I. Nevo, J. L. Hansen, and H. Stapelfeldt, J. Chem. Phys. 131, 064309 (2009).

[32] D. Davis, D. Shirley, and T. Thomas, J. Am. Chem. Soc. 94, 6565 (1972).

[33] G. A. Garcia, L. Nahon, and I. Powis, Rev. Sci. Instrum. 75, 4989 (2004).

[34] M. Yamazaki, J.-i. Adachi, Y. Kimura, M. Stener, P. Decleva, and A. Yagishita, J. Chem. Phys. 133, 164301 (2010).

[35] M. Stener, P. Decleva, J. Adachi, N. Miyauchi, M. Yamazaki, and A. Yagishita, J. Phys. B 45, 194004 (2012).

[36] T. Mizuno, J. Adachi, N. Miyauchi, M. Kazama, M. Stener, P. Decleva, and A. Yagishita, J. Chem. Phys. 136, 074305 (2012).

[37] See Supplemental Material at http://link.aps.org/supplemental/ 10.1103/PhysRevA.88.061402 for the ratio between the $\mathrm{F}(1 s)$ partial ionization cross sections of one-dimensionally aligned and randomly oriented $\mathrm{pFAB}$ molecules as a function of photon energy.

[38] M. Stener, S. Furlan, and P. Decleva, J. Phys. B 33, 1081 (2000).

[39] S. Schorb et al., Appl. Phys. Lett. 100, 121107 (2012).

[40] M. Harmand et al., Nat. Photonics 7, 215 (2013). 